\title{
Musikalische Innovation im Umfeld der Moderne und historischen Avantgarde in Ungarn
}

\author{
ANDREA VAN DER SMISSEN, WIEN
}

In Ungarn haben in den vergangenen Jahrzehnten mehrere Faktoren die musikhistoriographische Interpretation der Zwischenkriegszeit bestimmt. Zum einen haben die kulturpolitischen Richtlinien der 1950er Jahre, die aus ideologischen Gründen einige progressive Kunstexperimente der vorangehenden Jahrzehnte ablehnten, eine nachhaltige Wirkung hinterlassen. Zum anderen besetzte die Nationalschule der modernen ungarischen Musik in ihrer Selbstdarstellung die 1920er und 1930er Jahre als den Zeitraum ihrer Formierung, weshalb angenommen werden kann, dass die Vereinnahmung dieser Periode mit der Vernachlässigung bestimmter musikhistorischer Fakten einhergeht. Wenn beispielweise György Kroó den "patriotischen Gemeinschaftscharakter"1 der modernen ungarischen Musik von Kodály hervorhebt, deutet er auf eine Tendenz der 1930er Jahre hin. Es ist jedoch irreführend, ausschließlich auf einer Tendenz der Nationalisierung der modernen Musik zu beharren und die parallelen Phänomene in der Kunst, wie auch deren internationales Widerspiel in der Zwischenkriegszeit nicht zu beachten. Der Literaturwissenschaftler Mihály Szegedy-Maszák kritisiert Judit Frigyesis aktuelle Bartók-Studie, $^{2}$ aus einem kulturgeschichtlichen Blickwinkel: Sie spiegele nicht einmal annähernd das intellektuelle Leben der frühen Zwanzigerjahre in Budapest wider und vereinfache damit die Rolle, die Bartók in seinem kulturellen Umfeld spielte. ${ }^{3}$ Dabei ist zu bemerken, dass die immer wieder herausgehobenen nationalen Merkmale von Bartóks Musik nur auf einen, für Bartóks Kunst nicht wesentlichen kultursoziologischen Aspekt des Nationalismus als möglichen geistesgeschichtlichen Hintergrund seiner Werke hindeuten.

Ein grundlegender und am meisten vernachlässigter Aspekt dieser Periode sind die kulturellen Verbindungen Ungarns zum restlichen Europa, die auch nach dem Zerfall der Monarchie intakt und mehrschichtig geblieben sind. ${ }^{4}$ Diese wurden durch die Internationalität der Avantgarde und Moderne mit neuen Anbindungen an transkulturellen Entwicklungen sogar intensiviert. Die Kulturregion ist anderseits aus kunstgeschichtlicher Sicht in den 1920er und 1930er Jahren durch die starke Präsenz einer ästhetischen Forderung nach ,Neuer Kunst' zu charakterisieren, die durch die wichtigsten Vertreter der Avantgarde und Moderne wie Lajos Kassák, Georg Lukács und Valéria Dienes zu bedeutenden Innovationen auf den Gebieten Literatur, bildender Kunst,

1 György Kroó, Ungarische Musik gestern und heute, Budapest 1981, S.43-44.

2 Judit Frigyesi, Béla Bartók and Turn of the Century Budapest, Berkeley 1998.

3 Mihály Szegedy-Maszák, "Bartók's place in Culture history”, in: Studia Musicologica Academiae Scientiarum Hungaricae 41, H. 4 (2000), S.457-465.

4 Pál Deréky, Ungarische Avantgarde Dichtung in Wien 1920-1926, Wien 1991, S.122. 
Theater und Bewegungskunst führten. Die kulturgeschichtlichen ${ }^{5}$ und kunstgeschichtlichen Kontexte der Zwischenkriegszeit implizieren die Vorstellung, dass eine musikalische Innovation im Rahmen einer transkulturellen Auffassung der Neuen Musik in Ungarn gegeben war.

Die Untersuchung der "Vereinigung Moderner Ungarischen Musiker" konnte diese Annahmen bestätigen. Die Gruppierung - im Folgenden ,MoMaMu'6 - trat erstmals im Jahr 1926 an die Öffentlichkeit.7 Ihre Mitglieder Pál Kadosa, György Kósa, Ferenc Szabó und István Szelényi repräsentierten der Forschung zufolge bis in die 1930er Jahre ihre gemeinsamen künstlerischen Interessen. ${ }^{8}$ Sie zeigten sich "rückwärtsgewandten Tendenzen" ${ }^{9}$ wie dem Neoklassizismus abgewandt ${ }^{10}$ und betonten die Notwendigkeit einer neuen Musikästhetik, die den Primärelementen der Musik eine neue Deutungsmöglichkeit über die Moderne hinaus eröffne. ${ }^{11}$

In den Biographien von Kadosa, ${ }^{12}$ Szelény $i^{13}$ und Szabó ${ }^{14}$ wird stets darauf hingewiesen, dass diese Komponisten Kontakte zu der ungarischen literarischen und bildkünstlerischen Avantgarde hatten. Über Kósa ist bekannt, dass er in einem intensiven künstlerischen Austausch mit der modernen Tanzbewegung stand. ${ }^{15}$ Durch eine Rekonstruktion der intermedialen und interkulturellen Netzwerke kann man auf die Rolle der Verknüpfungen zur Avantgarde und Moderne für die künstlerische Orientierung der Komponisten der MoMaMu schließen. Diese waren soweit maßgebend, dass sie nicht nur zur Übernahme der Vorreiterrolle in den musikalischen Zielsetzungen der Komponisten prädestinierten, sondern darüber hinaus zur Entlehnung außermusikalischer Konzepte bei der Herstellung neuer kompositorischer Entwürfe beitrugen. Die Gründung der Vereinigung MoMaMu ist vermutlich mit den veränderten Rezeptionsbedingungen der Neuen Musik und mit der Institutionalisierung ihrer Foren in der ersten Hälfte des 20. Jahrhunderts verbunden. Im Zentrum des musikwissenschaftlichen Interesses stehen jedoch die neue Kontextualisierung von Werken der Komponisten und die Erforschung der musikalischen Innovation, die über die Moderne und die bekannten musikalischen

5 Zu erwähnen ist auch die Zweisprachigkeit der Komponisten, wobei keine erlernten Sprachfähigkeiten gemeint sind, sondern die Mehrsprachigkeit der Bevölkerung der ehemaligen Länder der Monarchie.

6 Abkürzung der ungarischen Bezeichnung ,Modern Magyar Muzsikusok Egyesülete'.

7 Öffentlicher Brief des Vorbereitungskomitees der ,MoMaMu' an das Kultusministerium, in: Új föld I, Nr. 1 (Februar 1927.) S. 30-31.

8 Gemeint ist u. a. die gemeinsame Repräsentation ihrer Werke. Siehe: Andrea van der Smissen, The Connection between Henry Cowell's New Music Society and Hungarian Composers of the Young Generation in the Twenties, Hungarian Academy of Sciences, Archives for 20 ${ }^{\text {th }}-21^{\text {st }}$ Century Hungarian Music, 2019.

9 Pál Kadosa, „Abschlussrede zu einer Aufführungsserie von moderner Musik an der Fodor-Musikschule”, HS. undatiert, pp.12. in: Kadosa-Nachlass, Archiv der Ungarischen Musik des 20-21. Jhs., MTA-BTK-ZTI.

10 Pál Kadosa und Ferenc Szabó änderten ihre künstlerische Einstellung in den 1930er Jahren.

11 István Szelényi, „Über den Sieg der [musikalischen] Reaktion”, in: Crescendo 1, H. 11-12 (Juni/Juli 1927), S.1-6.

12 Vgl. János Breuer, Tizenhárom óra Kadosa Pállal [Dreizehn Stunde mit Pál Kadosa], Budapest 1978, S. 38.

13 Zu István Szelényi gibt es keine biographische Literatur.

14 Vgl. János Maróthy, Zene, Forradalom, Szocializmus. Szabó Ferenc útja [Musik, Revolution, Sozialismus. Der Weg Ferenc Szabós], Budapest 1975, S. 30.

15 Vgl. Anna Dalos, „Életpálya” [Lebensweg], in: György Kósa, hrsg. von Melinda Berlász, Budapest 2003, S. 11-44, hier S. 16. 
Strömungen hinausreicht. Dabei ist die Betrachtung einzelner musikalischer Werke aus analytischer Sicht oft problematisch. Es scheint so, dass das notwendige analytische Instrumentarium aus den neu gewonnenen Zusammenhängen mit anderen stilistisch und kompositionstechnisch vergleichbaren Werken oder ästhetischen Vorgaben, die in diesem Fall auch nichtmusikalisch sein können, abzuleiten sind. Im Folgenden wird weniger auf eine stilistische Zuordnung und Beurteilung der ästhetischen Qualität der exemplarisch ausgewählten Kompositionen der Komponisten der MoMaMu Wert gelegt als auf die Darstellung des möglichen Auftretens und der Art kompositionsgeschichtlicher Durchbruchsphänomene.

\section{György Kósa: Sechs Orchesterstücke (1919)}

Kósas Sechs Orchesterstücke wurde im Jahr 1925 bei dem Prager Musikfestival der Internationalen Gesellschaft für Neue Musik (IGNM) von Václav Talich bzw. von Erich Kleiber ${ }^{16}$ in Berlin aufgeführt. ${ }^{17}$ Auf ihren Erfolg hin erhielt er einen Exklusivvertrag mit der Universal Edition (UE) in Wien. Die Originalform der Komposition wurde als Sechs Klavierstücke (1919) im Jahr 1926 herausgegeben. Theodor W. Adorno kritisierte die "als Miniaturen zu wenig konzentrierten Stücke, in denen die Themen die Dimensionen sprengen" und deren "harmonisch und kontrapunktisch oberflächliche" Ausarbeitung. ${ }^{18}$

Die analytischen und interpretatorischen Grundlagen der Sechs Orchesterstücke verbergen sich in der intermedialen Verbindung von Kósas Musik zu der am Schnittpunkt der Lebensreformbewegung und der experimentellen Moderne stehenden Bewegungskunst von Valéria Dienes. Auf die enge Zusammenarbeit des Komponisten mit Dienes, deren Orkestik-Konzept die Bewegungsprinzipien des menschlichen Körpers ${ }^{19}$ als eine evolutive Einheit unterschiedlicher disziplinärer Blickwinkel ${ }^{20}$ erfasste, lässt sich zurückführen, dass die Komposition auf der Grundlage des theoretischen Systems der Bewegungskunst - zu der auch eine neue, eng aufeinander bezogene Musik-Tanz-Verbindung gehörte - auf nicht-musikalisch inspirierte Idiomatik und Formprinzipien aufbaut.

16 Die Aufführung fand am 14. März in der Berliner Staatsoper mit der Berliner Staatskapelle statt. Erich Kleiber galt als Förderer Kósas, sie trafen sich mehrmals in Budapest und in Berlin zu Konsultationen.

17 Unter der Leitung von Ernö Dohnányi kam das Werk in Budapest am 4. Dezember 1922 und in New York am 12. Dezember 1926 zur Aufführung.

18 Theodor W. Adorno, „Rezension zu Sechs Klavierstücke. Bagatelle für Klavier”, in: Die Musik 19 (2. Hj 1927), S. 604.

19 Auf ähnlichen Grundlagen baut die konduktive Methode von András Petö.

20 An den Grenzen der Kunst und Wissenschaft, Tanz und Philosophie. Siehe: Márk Fenyves und Brigitta Balogh, „Bevezetés Dienes Valéria terminológiájába" [Einführung in Valéria Dienes' Terminologie], in: Kellék, H. 60 (2018), S. 39-60, hier S. 39. 
In Ungarn wurde durch Lukács antipositivistische Sichtweise ${ }^{21}$ und Dienes' Bergsonismus ${ }^{22}$ die Kunstdiskussion über die Moderne durch Begrifflichkeiten - welche künstlerische Inhalte und Haltung definierten - wie Mikrokosmos ${ }^{23}$ und Rückzug in die Innerlichkeit geprägt.. ${ }^{24}$ Für Dienes und Kósa wurde der Mikrokosmos des Individuums, eine in ihrer ursprünglichen Gegebenheit nicht vermittelbare innere Welt, der Mittelpunkt der künstlerischen Arbeit, die mit einer miteinander geführten "Instrospection" verbunden war. ${ }^{25}$ In Dienes' Kunstkonzept, das in der Interpretation von Brigitte Balogh im weitesten Sinne mit einer Erkenntnistheorie gleichgestellt werden kann, ${ }^{26}$ spielt Henri Bergsons Verständnis des "Conscience" eine zentrale Rolle. ${ }^{27}$ Um auf die nicht-bewussten Vorgänge, die in der menschlichen Bewegung mitresonieren, aufmerksam zu machen, ${ }^{28}$ mied sie den von der Psychologie besetzten Begriff des ,Bewusstseins'. Ebenso wurde in Dienes' System das Grundelement des Tanzes - die "Bewegung" oder "mozdulat" $^{\prime 29}$ - durch die von Bergson in Matière et mémoire ${ }^{30}$ beschriebenen Ansätze geprägt. Wenn Kósa in Sechs Orchesterstücke Bewegungen nachzeichnet, folgt er Henri Bergsons Verständnis vom Gedächtnis, gekennzeichnet durch die Signifikanz der Verbindung der durchlebten Vergangenheit und der körperlichen Gegenwart.

DieSätze der Komposition- „Einsamkeit", , Galgenhumor", ,"Gebet des Zweiflers", , Schüchterne Sehnsucht", "Justament", „Hoffnungslos" - als Ergebnis rhythmisch-analytischer Verarbeitung relevanter Bewegungsreihen aus „mimischen Studien“31 bauen auf rhythmischen, agogischen

21 Gemeint ist Georg Lukács philosophische Orientierung vor 1918 und sein Einfluss auf die Theoretiker des Sonntagkreises.

22 Valéria Dienes übersetzte die Werke Henri Bergsons ins Ungarische und ermöglichte so die Rezeption seiner Lebensphilosophie in der Zwischenkriegszeit. Bergsons Sicht auf die metaphysische Einheit des Lebens und seine intuitive Erkenntnistheorie veranlassten Dienes unter anderem über Schulpädagogik nachzudenken. Siehe: Valéria Dienes, "Bergsonismus in der Schule”, in: Nyugat 17 (1924), <https://epa.oszk.hu/00000/00022/nyugat. htm> (24.09.2020).

23 Lukács definiert den Mikrokosmos als nicht-psychologierende Beschreibung des menschlichen Innenlebens als Folgerung aus der Theorie der neukantianischen Gegenstandkonstitution. Siehe: Konstantin Kavoulakos, Ästhetische Kulturkritik und ethische Utopie. Georg Lukács' neukantianisches Frühwerk, (Deutsche Zeitschrift für Philosophie, Sonderband), Berlin 2014, S. 79.

24 "Die These einer Trennung der objektiven ,Zivilisation' und subjektiven ,Kultur' war innerhalb der deutschen Kulturphilosophie und Sozialtheorie verbreitet." Kavoulakos, Ästhetische Kulturkritik, S. 17.

25 Mária Mirkovszky, „Dr Dienes Valéria elöadásai az Orkesztika köréböl. Jegyzetek”, in: Dienes-Nachlass, MOHA. Siehe auch: Valéria Dienes, „Az elsö valóságelelm” (1974), in: A tánc reformja a mozdulatmüvészet vonzásában [Tanzreform im Vorfeld der Bewegungskunst], hrsg. von Márk Fenyves, Valéria Dienes und Gedeon Dienes, Budapest 2016, S. 115-119, hier S. 115.

26 Brigitte Balogh, „A szerkesztö elöszava“ [Vorwort zur Ausgabe] Valóság-mozdulat-gondolat. Dienes Valéria filozófiája, in: Kellék, H. 60 (2018), S. 7-10.

27 Dies geht auf die Rezeption von Bergsons Essai sur les donées immédiates de la concience (Paris 1889) zurück. Bergsons erkenntnistheoretische Ansätze über das nichtbegriffliche Denken beeinflussten nicht nur Dienes, sondern auch die Theoretiker des Sonntagskreises wie etwa Arnold Hauser.

28 Márk Femnyves und Brigitta Balogh, „Bevezetés Dienes Valéria terminológiájába“ [Einführung in Dienes Terminologie], in: Kellék, H. 60 (2018), S. 39-60, hier S. 40.

29 Ungarisch; es bedeutet so viel wie Positionswechsel des Körpers innerhalb einer Bewegungsreihe oder Bewegungseinheit.

30 Henri Bergson, Matière et mémoire. Essai sur la relation du corps à l'esprit, Paris 1896.

31 György Kósas weitere Kompositionen wie die 13 Bagatelle (1919) sind nach der Definition Dienes' mimische Studien. 
und die Melodie bestimmenden Elementen, die aus dem Ineinanderreflektieren der korrelierenden zeitlichen, dynamischen, inhaltlichen und räumlichen ${ }^{32}$ Komponenten beider Gattungen - Tanz und Musik - erarbeitet wurden, auf. Auch die Kontrastierung der musikalischen Elemente und die inhaltliche Verdichtung kann aus den tänzerischen Entsprechungen, z. B. der Nachzeichnung der Steigerung und des Abfallens von kinetischen Energien oder aus einem Bewegungskanon interpretiert werden.

Kósas Musik ist ein besonderes Beispiel für einen Anspruch auf die dem Kunstwerk vorausgesetzten Konzepte der modernen Musik sowie für deren ,esoterische' Sensibilisierung.

\section{Einfluss des Konstruktivismus in den Werken der Komponisten der MoMaMu}

Die literarische und bildkünstlerische Avantgarde vermittelte ideologisch weitgehend anders gestützte künstlerisch-ästhetische Konzepte. István Szelényis musikalische Schriften berufen sich auf Lajos Kassáks Aktionismus und Konstruktivismus ${ }^{33}$ und darüber hinaus auf Viking Eggelins Konzept des absoluten Films, ${ }^{34}$ wenn er seine Definition von einer absoluten Musik festlegt, die durch wissenschaftliche, exakt definierte, abstrakte ästhetische Grundelemente und deren neu definierten Gesetzmäßigkeiten verwirklicht wird. ${ }^{35}$ Szelényis Zielsetzung war, affektive und geistige Wertigkeiten wie auch das Naturalistische in der Musik zu vermeiden. ${ }^{36}$ Bei der Übernahme von Kassáks Kunstanschauung reflektiert er über die mit dem russischen Konstruktivismus vergleichbaren Ansätze, nach denen die Kunst eine zentrale Handlungsinstanz in der Gesellschaft ist, $d$. h. sie beeinflusst sie nicht nur, sondern konstruiert eine neue Realität. ${ }^{37}$

Szelényis Klavierstück Manifestum, Sonata alla marcia (September 1928), ist eine aktivistische $^{38}$ Anspielung auf eine getragene Marsch-Musik, deren nach oben steigende Phrasen Energie vermitteln. Die wenigen eingesetzten Motive treten nebeneinander oder ineinander verschoben in der Komposition auf, und ähnlich wie im Falle einer Collage kommt es nicht zu ihrer Weiterentwicklung oder Permutation. Szelényi komponiert stellenweise den Zerfall der zwei aufeinander bezogenen Stimmen in der linken und rechten Hand aus, indem beide sich metrisch unabhängig voneinander bewegen, um dann wieder zur strengen rhythmischen Organisation der Stimmen zurückzukehren. Um die Verbundenheit der Stimmen zu stärken,

32 Der vertikale Aufbau der Musik wurde mit räumlichen Elementen des Tanzes in Korrelation gesetzt.

33 István Szelényi, „Die wichtigsten Strömungen der modernen Musik”, in: Crescendo 2, H. 6-7 (Januar/Februar 1928), S. 3-11.

34 Ebd.

35 Ebd.

36 Ebd., S. 5.

37 Ebd., S. 11

38 Ebd., S. 11. Vgl. mit Szelényis Äußerung: „Die Neue Kunst ist Aktion!“ Der Aktionismus ist eine der linken Arbeiterbewegung nahestehende avantgardistische Bewegung. Szelényi übernimmt jedoch nicht Kassáks linke Orientierung, er bezieht sich auf die Aufforderung zur Ablehnung eines "passiven Vorbeiempfindens" der Gegenwart. 
werden dialogisierend musikalische Ereignisse und Motive voneinander übernommen und ,addiert', so dass diese - für die Analyse erkennbar - der rechten oder linken Hand in ihrer ursprünglichen Form zugeordnet werden können. Szelényi hat sich in Manifestum ähnlich, wie in seinen Kompositionen seit 1926, auf eine streng dissonante Tonsprache festgelegt, er verwendet ausschließlich Sekunden-, Septimen- und Tritonus-Intervalle. Die Akkordbildung drängt - vom Marsch-Motiv getragen - Richtung Cluster-Bildung, die aus, bis zu fünf aufeinander gebauten Sekunde bestehen. Die harte Klangwelt des Stückes wird durch auseinanderliegende Klangfarben harscher, wenn in der weiten Lage die äußersten Register des Klaviers Verwendung finden. ${ }^{39}$

Die Serenade ${ }^{40}$ für Bratsche und Flöte (1926) von Ferenc Szabó zeigt besonders in ihrem ersten Satz eine ähnliche Zuwendung zur konstruktivistischen Aufstellung des musikalischen Materials und der Form. ${ }^{41}$ Das Werk lässt seinen experimentellen Charakter dann erkennen, wenn man es im Kontext seiner Uraufführung sieht. Die frühe Fassung des Stücks wurde am 23. Oktober $1926^{42}$ als Begleitmusik zu Aladár Tamás' Gedicht „Der Wind schreitet auf Glasbeinen" 43 das erste Mal aufgeführt, wobei an der künstlerisch mehrschichtigen Produktion ein Sprechchor und die Bewegungskünstlerin Magda Förstner beteiligt waren. Performances, die auf der Verbindung von Gedicht und Bewegungskunst basierten, kamen bei den historischen Avantgarde-Vorstellungen sehr häufig vor, die Verwendung einer modernen Komposition war jedoch selten. Die durch Septimen überladene Klangwelt der Komposition hatte für Szabó vermutlich eine psychoakustische Bedeutung, da er das Stück in mehreren Besetzungen ausprobierte. $^{44}$

Die Serenade besteht aus drei Sätzen, wobei jeder Satz eine andere Gestaltungsidee in insgesamt 151 Takten verwirklicht. ${ }^{45}$ Der erste Satz besteht aus 50 Takten und bekommt quasi die Form einer Sonate, die monothematisch gestaltet ist. In den ersten zwei Takten werden diejenigen musikalischen Elemente präsentiert, auf denen der Satz aufgebaut wird: $d$, als Ausgangs und Schlusston des Satzes ohne tonale Bezüge, die e-f-e-Sekundschritte und darauffolgend zwei reine Quartschritte abwärts bzw. eine reine Quinte darauffolgend aufwärts. Die Themeneinsätze in der Flöte- und Bratschenstimmen erfolgen mit zwei Takten Unterschied und im Quintabstand. Es scheint so, dass der Satz auf dem Antagonismus der Dissonanz und Konsonanz aufgrund der Sekunde, des Tritonus und der Septime und der reinen Intervalle,

39 Wie auch die 2 Recitative (1926) und 3 Skizzen (1926-1927), die in der Zwischenkriegszeit häufig aufgeführt wurden.

40 Die Komposition wurde posthum entdeckt und erfuhr in den 1950er und 1960er Jahren keine Umarbeitung.

41 Als musikalische Terminologie wird Konstruktivismus von Peter P. Várnai verwendet. Siehe: Peter P. Várnai, "István Szelényi" in: Grove Dictionary of Music, Bd.24, S.879-880.

42 Avantgardistischer Präsentationsabend der Zeitschrift Új Föld [Neues Terrain]. Als eigenständige Komposition wurde die Serenade am 9. April 1927 an einem weiteren Új-Föld Abend aufgeführt.

43 Aladár Tamás, Üveglábakon jár a szél. (Die Übersetzung A.v.d.S.).

44 Siehe die Flöte-Geige-Version und die 3 Klavierstücke Op. $5 a$.

45 Die einzelnen Sätze sind nach der Taktanzahl unterschiedlich lang. 
als konstruktivistisches Prinzip aufgebaut ist, das nicht nur die Stimmabläufe vertikal, sondern auch horizontal bestimmt; durch den Quintabstand zwischen den beiden Stimmen entstehen gehäuft Septimenzusammenklänge, die das Klangbild des Satzes dominieren. Das Thema wird im zwölften Takt eine große Sekunde höher wiederaufgenommen, zuerst von der Bratschenstimme, dann zwei Takte versetzt von der Flöte, worauf eine kurze Ausarbeitung folgt. In der Reprise werden die ersten zehn Takte des Satzes in Originaltonhöhe wiederholt. Die abschlieBende Coda besteht ebenfalls aus zehn Takten.

Als gemeinsame Merkmale der Kompositionen von Szelényi und Szabó, die einen konstruktivistischen kompositorischen Ansatz aufweisen, kann man die variablen kompositorischen Ideen, die einerseits auf den jeweils definierten Gesetzmäßigkeiten zur Organisation des musikalischen Materials und anderseits auf der Meidung der musikalischen Idiomatik ausgehen, nennen.

\section{István Szelényi: Simultanfonie}

Das kammermusikalische Werk Simultanfonie von István Szelényi wird im Artikel zu István Szelényi in The Grove Dictionary of Music and Musicians nicht erwähnt. ${ }^{46} \mathrm{Im}$ Artikel von Rainer Mohrs in der Musik in Geschichte und Gegenwart ${ }^{47}$ wird es ohne Szelényis Originaltitel als Quintett für Klavier und Streicher mit dem Entstehungsjahr 1931 angegeben. ${ }^{48}$

Am Anfang der 1930er Jahre verwirklichte Szelényi hauptsächlich während seines Aufenthalts in Paris eine Reihe von innovativen kompositorischen Ideen, wie En avant _ Overture activiste (1930), Konstruktion für Klavier und Streichorchester (1931), Progressiv und Primitiv (1932), Supersong (1932) und Thematic for Cello (1932). Geht man von der Möglichkeit aus, dass bei der Entstehung der Simultanfonie Einflüsse der Musik von Henry Cowell wirksam wurden ${ }^{49}$ - die sich seit 1926 in Szelényis Kompositionen nachweisen lassen -, wäre ein späteres Entstehungsjahr denkbar. So datiert András Wilhelm das Werk auf 1938. ${ }^{50}$ Mit diesem Entstehungsdatum würde die Komposition zwischen Cowells 3. Streichquartett Mosaic (1935) und der Amerind Suite (1939) fallen..$^{51}$ In der Simultanfonie weist neben der "Indeterminacy" ${ }^{42}$ als

\footnotetext{
46 Peter P. Várnai, "István Szelényi”, in: The Grove Dictionary of Music and Musicians, Bd. 24. S.879-880.

47 Reiner Mohrs, Art. „István Szelényi“, in: MGG, Bd. 16, S. 384.

48 András Wilhelm datiert das Werk auf das Jahr 1938. András Wilhelm, „,István Szelényis Werke'. Werkverzeichnis, Typoskript, unveröffentlicht, 1977", in: Szelényi-Nachlass, im Besitz von Cornelia Szelenyi.

49 van der Smissen, The Connection.

50 Wilhelm, „István Szelényis Werke" .

51 David Nicholls, "Getting rid of the Glue: The Music of the New York School", in: American Art and Music. Journal of American Studies 27, H. 3 (Dezember 1993), S. 335-353, hier S. 351.

52 Ebd., S. 352.
} 
kompositorische Idee auch die Anlehnung des Tonvorrats im Motto-artigen Hauptthema an die Weltmusik ${ }^{53}$ auf Tendenzen hin, die bei Cowell zu beobachten sind. ${ }^{54}$

Die Simultanfonie folgt Szelényis Konzept einer konstruktivistischen absoluten Musik. Der Komponist fokussiert sich auf eine Auffassung der Musik als Summe "akustischer, zeitlicher" Abläufe. ${ }^{55}$ Im Vergleich zu früheren Werken gelingt ihm ein deutlicher Fortschritt, wenn es um die Erneuerung der musikalischen Sprache geht. Er schreibt den Instrumenten des Quintetts metrisch unterschiedliche offene Abläufe im Stimmverband ohne kontrapunktische Bestimmung vor, die in freie Improvisation mit vorgegebenem musikalischem Material übergehen. Die Einbringung der Simultanität hat für das Werk neben der neuen experimentellen Anwendung eines aleatorischen Verfahrens in einer weiteren gestalterischen Dimension Bedeutung. Die Anordnung von unterschiedlichen akustischen Linien führen unvermeidlich zu einer hörpsychologischen Überlastung, der Szelényi ebenfalls Kontrast-Momente bietet. Die kakophonischen Energien in der Musik werden durch klangliche Entspannungsphasen abgelöst. Auch die repetitive Verwendung eines minimalen Tonvorrats, der in einem wesentlichen Teil der Komposition als kompositorische Idee eingesetzt wird, wird in dieser hörpsychologischen Dimension der Musik wirksam.

Alexander Jemnitz kommentiert in der Tagespresse anlässlich der Uraufführung (1938) die „Parallelität in der zeitlichen und räumlichen Zuordnung" ${ }^{45}$ als Inhalt der Komposition. Er bringt damit das Werk mit dem philosophischen Diskurs in Zusammenhang, der anhand Henri Bergsons Werk Dureé et Simultaneite ${ }^{57}$ die neue Zeitmodellierung und das Erlebnismoment der neu erfahrenen Begrenztheit der Erkenntnisfähigkeit des einzelnen modernen Menschen thematisierte. ${ }^{58}$ Szelényi bezieht sich in seiner ideengeschichtlichen Arbeit ${ }^{59}$ auf die für die ungarische geisteswissenschaftliche Schule charakteristische Verbindung von Musikgeschichte und Geistesgeschichte ${ }^{60}$ und nimmt zugleich Bezug auf Bergsons Erkenntnistheorie. Er meint, dass die Bestandsaufnahme von Ansichten und Urteilen immer nur momentan und zufällig sei und nur

53 Der Tonvorrat $g$-c-des- $g$ ' des Motto-Themas ist eine Adaptation eines Teils der Gamelan Pelog-Skala. Auch die vierfache Oktavierung des Themas deutet auf den Einfluss der Gamelan-Musik.

54 Elliott Antokoletzt, A History of Twentieth-Century Music in Theoretic-Analytical Context, New York/Abingdon 2014, S. 164.

55 István Szelényi, „Die wichtigste Strömungen der modernen Musik”, in: Crescendo 2, H. 6-7 (January/February 1928), S. 3-11, hier S. 5.

56 Alexander Jemnitz, „Szimutánfónia”, in: Népszava 66, H. 110 (15. Mai 1938), S. 10.

57 Henri Bergson, Durée et Simultanéité. A propos de la théorie d'Einstein, Paris 1922.

58 Weist auf die Rezeption von u. a. Bergsons Essai sur les donées immédiates de la concience. Paris 1889 [Zeit und Freiheit (1911)] hin. Arnold Hauser, Mitglied des Sonntagskreises widmet in seinem Buch Sozialgeschichte der Kunst und Literatur im Kapitel über moderne Kunst dem Begriff Simultaneität ein Unterkapitel und weist dabei unmittelbar auf die Kunst und Kunstrichtungen der Zwischenkriegszeit hin. Arnold Hauser, Sozialgeschichte der Kunst und Literatur, Ungekürzte Sonderausgabe, München 1953, S. 999-1020.

59 István Szelényi, Zenetörténet és bölcsészettudomány kapcsolata [Die Verbindung der Musikgeschichte und Philosophiegeschichte], Budapest 1944.

60 Georg Lukács, Karl Mannheim und der Sonntagskreis, hrsg. von Eva Karádi-Erzsébet Vezér, Frankfurt a.M. 1985, S. 3. 
zu einer möglichen Erfassung verschiedener simultaner Lagedifferenzen führe. ${ }^{61}$ Seine Formulierung projiziert die Zufälligkeit des Moments, in dem sich mannigfache Relationen zueinander gruppieren.

Horst Bergmeier weist darauf hin, dass die Simultaneität in der Polemik der Ästhetik der historischen Avantgarde mehrfach enthalten ist. ${ }^{62}$ Die Befreiung des Kunstwerkes von Intention, die Idee, die John Cage als "die Klänge sind frei" ${ }^{\prime 63}$ formulierte, wurde in der historischen Avantgarde, in der Literatur und Bildenden Kunst zur Zeit Szelényis u. a. von Vertretern und Vertreterinnen des Dadaismus umgesetzt. ${ }^{64}$ Das futuristische Programm enthielt Filippo Tommaso Marinettis Ankündigung über „unsere anti-anmutige Musik ohne rhythmische Quadratur, unsere Geräuschkunst und unsere befreiten Worte". ${ }^{65}$ Cage, der auch als Cowells Schüler bezeichnet wird, verwendet den Ausdruck "getting rid of the glue"66 für den Zerfall des musikalischen Materials ${ }^{67}$ ab den 1950er Jahren und beruft sich dabei auf die wichtigen Impulse von Henry Cowell. ${ }^{68}$

Szelényis Werk nimmt die experimentellen Fortschritte der musikalischen Avantgarde zeitlich vorweg, wobei angenommen werden kann, dass dabei auch Cowells Intentionen ${ }^{69}$ über die Unbestimmtheit des musikalischen Materials in den 1930er Jahren ein Auslöser waren.

\section{Pál Kadosa: I. Klaviersonate Op.7 (1926)}

Kadosa schloss die Arbeit an seiner Klaviersonate Op.7 ungefähr zwei Monaten nach Cowells Besuch in Budapest ${ }^{70}$ im Juni/Juli 1926 ab. Die Uraufführung der Komposition fand Ende 1927 in Kalifornien statt ${ }^{71}$ und wurde anschließend bei den Konzerten der New Music Society ${ }^{72}$ und Pro Musica Society ${ }^{73}$ gespielt.

\footnotetext{
61 István Szelényi, Zenetörténet és Bölcselettudomány kapcsolata, S. 14-15.

62 Horst Bergmeier, Dada-Zürich: ästhetische Theorie der historischen Avantgarde, Göttingen 2011, S. 175.

63 David Nicholls, "Getting rid of the Glue: The Music of the New York School", S. 341.

64 Bergmeier, Dada-Zürich, S. 175.

65 Ebd.

66 Nicholls, "Getting rid of the Glue", S. 335-353.

67 Nicholls nennt dabei Cages Music of Changes (1951) als frühestes Beispiel.

68 John Cage, Silence: Lectures and Writings History of experimental music, Middletown/Connecticut 1961, S. 71.

69 Nicholls, "Getting rid of the Glue", S. 351.

70 Henry Cowell spielte sein Klavier-Recital am 28. April 1926 in Budapest, im großen Saal der Musikakademie. Kadosas Pianosonata wurde im Juni/Juli 1926 fertiggestellt und der Tänzerin Magda Förstner gewidmet.

71 van der Smissen, The Contact, S. 22.

72 Am 22., 28. und 30. November 1927. Am Sonntag, den 22. November 1927 fand das Konzert im Studio von Miss de Fremery (77 Fairmount avenue, Oakland), am 28. November 1927 im Studio von John Emmett Gerrity (2025 Durant Avenue, Berkeley) statt.

73 17. November 1927, Galerie Beaux Arts, San Francisco.
} 
Die in den 1920er Jahren von Cowells Kreis der "ultra modernen" Bewegung rezipierte Komposition ist in ihrer ursprünglichen Form ${ }^{74}$ gänzlich unbekannt. Von der Klaviersonate nimmt die Fachliteratur ausschließlich in der vierzig Jahre später umgearbeiteten, ${ }^{75}$ und im Jahr 1968 herausgegebenen Fassung, Kenntnis, ${ }^{76}$ wobei das Werk weiterhin mit dem Entstehungsjahr 1926 datiert wird.

John D. Spilker weist in seinem Buch The Origins of "Dissonant Counterpoint": Henry Cowell's Unpublished Notebook ${ }^{77}$ darauf hin, dass Cowell für die Verbreitung seiner Theorie sorgte: "Henry Cowell, however, continued to work on the method during the late 1920 and throughout the 1920s, and he even disseminated it to other composers. ${ }^{178}$ Zwischen Cowell und den Komponisten der MoMaMu bestand in der zweiten Hälfte der 1920er Jahre eine Arbeitsbeziehung, ${ }^{79}$ was dadurch belegt werden kann, dass Kadosa und Szelényi seit 1926 Mitglied des ,Advisory Board' von Cowells New Music Society waren. Im Rahmen der Zusammenarbeit wurden zwischen Kalifornien und Budapest aktuelle Nachrichten wie auch Kompositionen ausgetauscht.

Aus der Begleitnotiz zur Postsendung ${ }^{80}$ die Szelényi an Cowell nach Kalifornien mit Kadosas Klaviersonate Op. 7 schickte, erfährt man, dass Szelényi bestrebt war die kompositorische Methode "Dissonant Counterpoint" (DC) bei seiner 3. Klaviersonate anzuwenden. ${ }^{81}$ Die Klaviersonate von Kadosa empfahl er an Cowell als "very remarkable". 82

Während Szelényis III. Klaviersonate als verschollen gilt, ist im Falle der Originalversion von Kadosas Komposition die Verwendung der Regeln des DC nachweisbar. In den Themen der drei Sätze der Klaviersonate, 1. Allegro maestoso - 2. Molto Allegro - 3. Adagio, sowie in der ganzen Textur des Werkes fallen betonte Zählzeiten, dissonante Klänge und Intervalle auf, konsonante Klänge und Intervalle werden , aufgelöst'. ${ }^{3}$ Die Befolgung der Regel des DC führt stellenweise zu einer sehr dünnen Textur des gesetzten musikalischen Materials bei Kadosa. Akkordbildung

74 Die MS befindet sich in: Kadosa-Nachlass, Archiv der Ungarischen Musik des 20.-21. Jhs., MTA-BTK-ZTI. Ferenc Bónis erwähnt das Werk in seinem Werkverzeichnis nur als Manuskript. Siehe: Ferenc Bónis, Kadosa Pál, Budapest 1965.

75 Ebd.

76 In der Konzertdatenbank der MTA-ZTI taucht das Werk das erste Mal in einem Konzertprogram vom 20.12.1969 auf.

77 John D. Spilker, "The Origins of "Dissonant Counterpoint': Henry Cowell's Unpublished Notebook", in: Journal of the American Music 5, H. 4 (2011), S. 481-533.

78 Ebd., S. 484.

79 van der Smissen, The Connection.

80 Brief, István Szelényi an Henry Cowell, undatiert [vermutlich von Ende Januar 1927], in: Henry-Cowell Estate, Box 15. Folder 19, NYPL.

81 Ebd.

82 Ebd.

83 Nach den Regeln des von Cowell in den Jahren 1914-17 herausgearbeiteten "Dissonant Counterpoint”, der sich am traditionellen Kontrapunkt orientiert, finden in Kompositionen ausschließlich dissonante Intervalle Verwendung, konsonante Intervalle dürfen zur Abwechslung verwendet werden, wenn sie vorbereitet und wieder aufgelöst werden. 
mit mehr als drei Tönen wird vermieden, dabei werden die Töne gern in weiter Lage, sehr oft in den beiden Stimmen oktaviert gesetzt. Im ersten Satz, der eine Sonatenform aufweist, sind weitere Regeln des DC in den Takten 82-85 zu beobachten. Hier erfolgt eine Sequenzierung eines Motivs sieben Mal, jeweils eine große Sekunde höher. Die linke Hand bringt zum sequenzierten Motiv keine eigenständige Gegenstimme, sondern nur Dur-Dreiklänge, die sich zu den Motiven in der rechten Hand fügen. So ergeben sich wiederholt Akkordformationen, die aus einem Dur-Dreiklang und einer dissonanten Sekunde bestehen, ${ }^{84}$ wobei die Reibung jeweils zwischen dem Grundton des Akkords und der Sekunde entsteht. Dies entspricht der Regel, die Cowell in seinem unveröffentlichten Notizbuch über DC aufgezeichnet hat: dass die Dissonanz bei drei oder mehreren Stimmen entweder in der Bassstimme oder auf die Bassstimme bezogen sein soll. 85

Es würde zu weit führen, an dieser Stelle detailliert zu der im Jahr 1968 überarbeiteten Version der Klaviersonate Stellung zu nehmen. Durch die kontrapunktische Ausarbeitung, die optimierte Form, eine mildere Tonsprache und die typischen an Bartók erinnernden Stilmittel - etwa im Tonmaterial oder in Form von eng geführten, auf wechselnden kleinen und großen Sekunden basierenden volksliedhaften Motiven bzw. funktionaler Differenzierung im strukturellen Gewicht - gibt diese eine andere kompositorische Orientierung vor. Kadosas Original der Klaviersonate wiederum setzt den Komponisten vorübergehend mit Henry Cowells Kreis und dessen ,ultra moderner' Bewegung in Verbindung.

Pál Kadosas und Ferenc Szabós Kompositionen aus der Zwischenkriegszeit wurden größtenteils in den 1950er und 1960er Jahren umgearbeitet und herausgegeben. Bezüglich des unveröffentlichten und sehr umfangreichen Notenmaterials des Kósa- und Szelényi-Oeuvres aus der Zwischenkriegszeit steht man dem Problem gegenüber, dass diese, insbesondere die symphonischen Werke ${ }^{86}$, nicht ausgewertet worden sind. Szelényis Kompositionen sind für die Forschung ohne Ausnahme unbekannt - so sind bis zum Zeitpunkt dieser Untersuchung keine Studien oder Veröffentlichungen zu seinem Leben oder kompositorischen Schaffen erschienen. ${ }^{87}$

Zitation: Andrea van der Smissen, „Musikalische Innovation im Umfeld der Moderne und historischen Avantgarde in Ungarn", in: Freie Beiträge zur Jahrestagung der Gesellschaft für Musikforschung 2019, hrsg. von Nina Jaeschke und Rebecca Grotjahn (= Musikwissenschaft: Aktuelle Perspektiven. Bericht über die Jahrestagung der Gesellschaft für Musikforschung 2019 in Paderborn und Detmold, Bd. 1), Detmold 2020, S. 335-346, DOI: 10.25366/2020.75.

84 Die Art der Akkordbildung, in denen die Sekunde als "Colour Note" mit aus Terz und Sexte gebauten Klängen kombiniert wird, findet in den Kompositionen von Szelényi und Kadosa häufig Verwendung und kann dem in Henry Cowell's Buch New Musical Ressources beschriebenen zweiten System von Akkordformationen zugeordnet werden.

85 Henry Cowell's Notebook on Dissonant Counterpoint, page 1 recto, zit. nach Spilker, "The Origins”, S. 490.

86 Zu erwähnen sind u. a. György Kósas Klaviersymphonie, oder Der Mensch und das Weltall (1921-22) und die Werke von István Szelényi.

87 Eine Ausnahme bildet ein Artikel über Haubenstock Ramati und Paul Arma, in dem István Szelényi Erwähnung findet. Siehe: Primavera Gruber, „Musikalische Grenzgänger" in: Wiener Zeitung (2. Juli 2004), S. 4. 


\section{Abstract}

In recent decades the interpretation of music history of the interwar period was determined by factors which allowed only national or folkloristic approaches to modern music in Hungary. However, the composers of the group 'Modern Hungarian Musicians', connected to the forums of the New Music like the ISCM or Cowell's NMS, were committed to a transcultural view of musical innovation. Through intermedial connections between literary and fine art, they received non-musical impulses by modern and avantgarde movements. This paper makes an approach on their heterogeneous conception of music with the common sense, to set a renewal of the musical language as its goal.

\section{Kurzvita}

Andrea van der Smissen wurde in Budapest/Ungarn geboren. Sie studierte an der Ferenc Liszt Musikhochschule Gesang, bevor sie 2007 ein musikwissenschaftliches Studium an der Universität Wien abschloss. Zurzeit arbeitet sie an ihrem Dissertationsprojekt über die Musikgeschichte der Zwischenkriegszeit in Ungarn. Die Fertigstellung der Dissertation erfolgt voraussichtlich im Jahr 2020. 


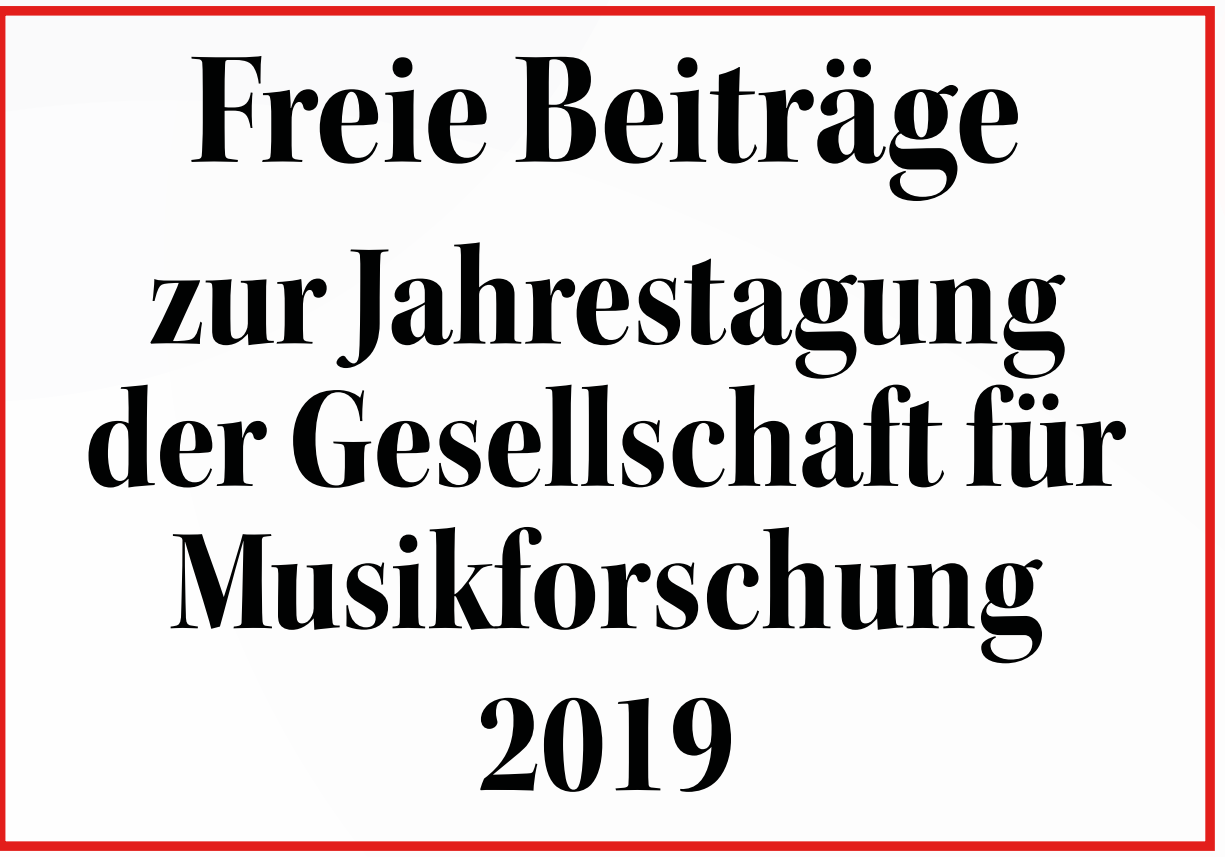

Herausgegeben von Nina Jaeschke und Rebecca Grotjahn

Musikwissenschaft: Aktuelle Perspektiven 1 
Freie Beiträge 


\section{Musikwissenschaft: Aktuelle Perspektiven}

Bericht über die Jahrestagung der Gesellschaft für Musikforschung 2019 in Paderborn und Detmold

Herausgegeben von Rebecca Grotjahn und Nina Jaeschke

Band 1 


\section{Freie Beiträge}

\section{zur Jahrestagung der Gesellschaft für Musikforschung 2019}

Herausgegeben von Nina Jaeschke und Rebecca Grotjahn

Detmold: Musikwissenschaftliches Seminar der Universität Paderborn und der Hochschule für Musik Detmold 2020 
DOI: $10.25366 / 2020.42$

Online-Version verfügbar unter der Lizenz: Urheberrecht 1.0, $<$ https://rightsstatements.org/page/InC/1.0/?language=de>

Bibliografische Information der Deutschen Nationalbibliothek

Die Deutsche Nationalbibliothek verzeichnet diese Publikation in der Deutschen Nationalbibliografie; detaillierte bibliografische Daten sind im Internet über http://dnb.d-nb.de abrufbar.

\section{Impressum}

Redaktion: Nina Jaeschke, Rebecca Grotjahn und Jonas Spieker Satz: Nina Jaeschke

(C) Musikwissenschaftliches Seminar der Universität Paderborn und der Hochschule für Musik Detmold 2020 


\section{INHALT}

Vorwort $\quad$ IX

Komponieren für das Radio: Akteure, Diskurse, Praktiken $\quad 1$

Musikwissenschaft - Feminismus - Kritik: Ein Generationenaustausch 6

\section{Stefan Alschner}

Der Wagner-Sänger Joseph Aloys Tichatschek - Vom Nachlass zum Netzwerk

\section{Alenka Barber-Kersovan}

Songs for the Goddess. Das popmusikalische Neo-Matriarchat zwischen Ethno-Beat,

erfundenen Traditionen und kommerzieller Vermarktung

Elias Berner, Julia Jaklin, Peter Provaznik, Matej Santi, Cornelia Szabó-Knotik

Musikgeschichte anders erzählen? Das Beispiel der 1970er in Österreich.

Musikhistoriographie in der Zeit der Digitalisierung

\section{Mauro Fosco Bertola}

„Ein Laut so klagevoll”. Lohengrin zwischen Richard Wagner und Salvatore Sciarrino

\section{Matthieu Cailliez}

Europäische Rezeption der Berliner Hofoper und Hofkapelle von 1842 bis 1849

\section{lacopo Cividini}

Zwischen klassischer Musikphilologie und angewandter Informatik:

Die Digitale Mozart-Edition (DME) der Stiftung Mozarteum Salzburg

\section{Marko Deisinger}

Fortschrittliche Technologie im Dienste eines Antimodernisten.

Heinrich Schenker und der österreichische Rundfunk

\section{Norbert Dubowy}

Vom Kritischen Bericht zur Kritischen Dokumentation am Beispiel der Digital-interaktiven Mozart-Edition

\section{Markus Engelhardt}

Musik zwischen Nation Building und Internationalität. Italien um 1900

\section{Maryam Haiawi}

Das Oratorium im Spannungsfeld der Konfessionen: 


\section{Judith I. Haug}

"Manch eine*r liegt, morgens noch trunken, im Rosengarten" - Rekonstruktionen

osmanischer Musikgeschichte in Gesangstextsammlungen

\section{Renate Koch}

Marcel Prawy und das erste Broadway-Musical im Österreich der Nachkriegszeit

Susanne Kogler, Julia Mair, Juliane Oberegger, Johanna Trummer

Erich Marckhl - Musikausbildung in der Steiermark nach 1945.

Brüche und Kontinuitäten

\section{Marie-Anne Kohl}

Die weinende Jury. "Geschlechtslose" Tränen bei globalen Musik-Castingshows?

\section{Fabian Kolb}

Tanztheater und filmische Ästhetik. Cineastische Einflüsse und Gestaltungsweisen in den Kompositionen für die Ballets Suédois 1920-1925

\section{Christian Lehmann}

Tempobezeichnungen von Julius Stockhausen für Die schöne Müllerin:

Ein Quellenfund

\section{Martin Link}

Signum et gens - Zur Gendersemiotik in Clara und Robert Schumanns Liederzyklus Liebesfrühling

\section{Livio Marcaletti}

„Strafspiel" und satirische Stilmittel in musikdramatischen Gattungen des frühen 18. Jahrhunderts

\section{Tobias Marx, Martin Lissner}

Thüringer Musikszene - Jugendmusikredaktionen als außerschulische musikbezogene Bildungskontexte

\section{Maho Naito}

Die Parallelität der Entstehungsprozesse der ersten beiden Symphonien Gustav

Mahlers: Instrumentation, Revision und Dirigierpraxis

\section{Elisa Novara}

Eine Schumann-Werkstatt? Zur Übertragbarkeit der Methoden vom Projekt 
Theodora Oancea, Joachim Pollmann, Jonas Spieker

Kollaborateure - Involvierte - Profiteure. Erarbeitung eines Online-Lexikons zur

Musik in der NS-Zeit

\section{Kiron Patka}

„Ich wollte eigentlich Sängerin werden." Berufsselbstbilder von Tontechniker*innen im Radio

\section{Siegwart Reichwald}

Die Leiden der jungen Clara: Das Klaviertrio Opus 17 als Ausdruck einer Neu-

Romantikerin

\section{Elisa Ringendahl}

Lied versus Oper - Pole musikalischer Gattungen bei Oscar Bie

\section{Benedikt Schubert}

Struktur und Exegese. Über Eigentümlichkeiten in der Arie "Des Vaters Stimme ließ sich hören" (BWV 7/4)

Uwe Seifert, Sebastian Klaßmann, Timo Varelmann, Nils Dahmen

Computational Thinking in der Musikwissenschaft: Jupyter Notebook als Umgebung

für Lehre und Forschung

\section{Yusuke Takamatsu}

Synthese als Modus der Prozessualität bei Schubert:

Sein spezifisches Wiederholungsprinzip im langsamen Satz

\section{Daniel Tiemeyer}

Johann Nepomuk Hummels Sonate in fis-Moll Op. 81 - Studien zu Entstehungs-

hintergrund, Rezeption und formalerStruktur

\section{Andrea van der Smissen}

Musikalische Innovation im Umfeld der Moderne und historischen Avantgarde in Ungarn

\section{Tim Ziemer, Holger Schultheis}

Psychoakustische Sonifikation zur Navigation in bildgeführter Chirurgie

\section{Magdalena Zorn}

Musik mit dem Radio hören: Über den Begriff der musikalischen Aufführung 
Gabriele Buschmeier in memoriam 


\section{Vorwort}

Die vorliegenden Bände dokumentieren die Jahrestagung der Gesellschaft für Musikforschung 2019. In den dreieinhalb Tagen vom 23. bis zum 26. September 2019 wurden in Paderborn und Detmold nicht weniger als 185 Beiträge präsentiert, verteilt auf diverse Symposien, Round tables, Freie Sektionen und Postersessions. Sie alle auf einen Nenner bringen zu wollen, ist ein Ding der Unmöglichkeit - und das ist gut so, ist es doch Ziel der Jahrestagungen, die große Vielfalt der Themen und Methoden des Faches Musikwissenschaft abzubilden. Um die thematische Vielfalt der freien Referate angemessen abbilden zu können und gleichzeitig den inhaltlichen Schwerpunkten der beiden hier publizierten Hauptsymposien ausreichend Raum bieten zu können, erscheinen diese in drei Bänden.

„Musikwissenschaft: Aktuelle Perspektiven": Der Titel der kleinen Reihe ist keine Verlegenheitslösung. Musikwissenschaft im Kontext der Digital Humanities; Musikwissenschaft und Feminismus; Musik und Medien; Musikalische Interpretation - schon die Felder, die von den vier Hauptsymposien bespielt wurden, wären noch vor wenigen Jahrzehnten allenfalls an der Peripherie das Faches zu finden gewesen. Sie entsprechen Arbeitsschwerpunkten der Lehrenden am Musikwissenschaftlichen Seminar der Universität Paderborn und der Hochschule für Musik Detmold, das die Tagung ausrichtete. Zugleich nehmen sie Bezug auf aktuelle Ereignisse und Entwicklungen. So erwuchs das von Andreas Münzmay und Joachim Veit organisierte Symposium „Brückenschläge - Informatik und Musikwissenschaft im Dialog" unmittelbar aus den Erfahrungen im Virtuellen Forschungsverbund Edirom (ViFE) und im fakultäten- und hochschulübergreifenden Zentrum Musik-Edition-Medien (ZenMEM). Der 200. Geburtstag von Clara Wieck/Schumann war der Anlass für das von Rebecca Grotjahn geleitete Symposium „Die Begleiterin - Clara Schumann, Lied und Liedinterpretation", das in enger Kooperation mit der Hochschule für Musik Detmold durchgeführt wurde. Das Hauptsymposium „Brückenschläge" wird in einem separaten Band publiziert (Bd. 3 der vorliegenden Reihe). Im Rahmen dieses Symposiums führte die von Stefanie Acquavella-Rauch geleitete Fachgruppe Digitale Musikwissenschaft eine Posterpräsentation durch, die von den Beiträger*innen erfreulicherweise zu kürzeren Texten umgearbeitet wurden, sodass sie hier ebenfalls, zusammen mit den Postern,

publiziert werden können. Hinzu kommen einige Beiträge, die bereits bei der Jahrestagung 2018 in Osnabrück präsentiert wurden. Auch das Hauptsymposium "Die Begleiterin" wird in einem eigenen Band (Bd. 2) publiziert. Die Beiträge zu den beiden anderen Hauptsymposien hingegen werden an anderen Orten veröffentlicht; in Band 1 („Freie Beiträge zur Jahrestagung der Gesellschaft für Musikforschung 2019") der vorliegenden Publikation finden sich jedoch Einführungen und Abstracts. Das Symposium „Komponieren für das Radio" unter Leitung von Antje Tumat und Camilla Bork (Katholieke Universiteit Leuven) behandelte Einflüsse des Mediums auf Kompositionsprozesse sowie durch radiophone Kompositionen bzw. radiophonen Klang ausgelöste Diskurse. Sarah Schauberger und Cornelia Bartsch (Universität Oldenburg) nahmen das 25-jährige Jubiläum der Fachgruppe Frauen- und Genderstudien zum Anlass für einen Generationenaustausch zum Thema "Musikwissenschaft - Feminismus - Kritik": Was wa- 
ren vor einem Vierteljahrhundert Inhalte und Aufgaben einer feministischen Musikwissenschaft und wie kann sich diese heute positionieren?

Bewusst haben wir im Tagungsbericht auf inhaltliche Eingriffe in die Beiträge verzichtet. ${ }^{1}$ Das gilt besonders für die Freien Referate: Es galt, den Charakter der Jahrestagung als Forum für ,freie', d. h. innovative und auch experimentelle Gedanken zu wahren. Einige Kolleg*innen, die die Tagung mit Vorträgen und Posterpräsentationen bereichert hatten, haben sich gegen eine Publikation im vorliegenden Band entschieden - sei es, weil sie eine Möglichkeit fanden, ihre Beiträge in einem inhaltlich passenderen Rahmen zu veröffentlichen, sei es, weil ihre Überlegungen in ihre entstehenden Qualifikationsschriften fließen sollen, oder sei es, weil sie von den Autor*innen in der vorgetragenen Form zunächst verworfen wurden. Auch damit erfüllt eine Freie-Referate-Sektion ihren Zweck: Die Diskussionen mit der versammelten Fach-Öffentlichkeit sollen dabei helfen, Gedanken weiterzuentwickeln und zu verändern. In diesem Sinne sei allen Beteiligten - den Autor*innen, den nichtpublizierenden Referent*innen und den Mit-Diskutant*innen - ganz herzlich gedankt für ihr Mitwirken bei der Tagung.

Unser herzlicher Dank gilt einer Reihe weiterer Personen, die zum Gelingen dieser drei Bände beigetragen haben. Hier ist besonders Jonas Spieker zu nennen, der uns tatkräftig bei der Redaktion geholfen hat. Andrea Hammes (SLUB Dresden) sei herzlich für die Aufnahme unseres Bandes auf musiconn.publish gedankt - wir freuen uns, damit unsererseits zur Etablierung dieser innovativen Publikationsplattform beizutragen.

Erneut möchten wir an dieser Stelle allen Menschen danken, die uns bei der Organisation, Ausrichtung und Finanzierung der Tagung selbst unterstützt haben: der Präsidentin der Universität Paderborn, Prof. Dr. Birgitt Riegraf, dem Rektor der Hochschule für Musik Detmold, Prof. Dr. Thomas Grosse, den Kolleginnen und Kollegen der beiden beteiligten Hochschulen, dem Vorstand der Gesellschaft für Musikforschung, der Universitätsgesellschaft Paderborn und allen Sponsoren. Besonders dankbar sind wir den Mitarbeiter*innen und den studentischen bzw. wissenschaftlichen Hilfskräften des Musikwissenschaftlichen Seminars, die bei der Vorbereitung und Ausrichtung der Tagung immensen Einsatz zeigten - stellvertretend sei an dieser Stelle Johanna Imm erwähnt, die zusammen mit Nina Jaeschke das Herz des Organisationsteams bildete.

Wir widmen diese Reihe Dr. Gabriele Buschmeier, dem langjährigen Vorstandsmitglied der Gesellschaft für Musikforschung, die kurz vor der Publikation dieses Bandes unerwartet verstarb.

Detmold, im September 2020

Rebecca Grotjahn und Nina Jaeschke

Zitation: Rebecca Grotjahn und Nina Jaeschke, „Vorwort”, in: Freie Beiträge zur Jahrestagung der Gesellschaft für Musikforschung 2019, hrsg. von Nina Jaeschke und Rebecca Grotjahn (= Musikwissenschaft: Aktuelle Perspektiven. Bericht über die Jahrestagung der Gesellschaft für Musikforschung 2019 in Paderborn und Detmold, Bd. 1), Detmold 2020, S. IX-X, DOI: 10.25366/2020.43.

1 Freigestellt war den Autor*innen auch, ob sie sich für eine gendersensible Sprache entscheiden bzw. welche Form des Genderns sie bevorzugen. 